(IDO1) expression was analysed by Western Blot and expression of enzymes of the kynurenine pathway by real-time PCR. Tryptophan/ kynurenine levels in culture supernatants were quantified by HPLC.

Results: SF strongly inhibited the proliferation of co-cultured Th cells. Tryptophan was completely depleted within a few days in co-cultures of SF and Th cells, resulting in eukaryotic initiation factor (eIF)2 $\alpha$ phosphorylation, TCR $\zeta$-chain downregulation and proliferation arrest. Blocking of IDO1 completely restored Th cell proliferation, indicating that SF suppressed the proliferation of Th cells through IDO1-mediated tryptophan catabolism. Interestingly, RASF showed a significantly lower IDO1 expression, tryptophan metabolism and a weaker Th cell suppressive capacity compared to OASF. Under hypoxic conditions, the secretion of IFNg, the expression of IDO1, the tryptophan metabolism and the Th cell suppressive capacity of both OASF and RASF were significantly reduced.

Conclusions: SF suppressed Th cell growth through IDO1-mediated tryptophan catabolism. This mechanism may play an important role in preventing inappropriate Th cell responses under normal conditions. The reduced tryptophan metabolism under hypoxia together with the inferior efficiency of RASF to restrict T cell proliferation likely supports the development of synovitis in RA.

Disclosure of Interest: None declared

DOI: 10.1136/annrheumdis-2017-eular.5146

\section{AB0069 STRONG AGE-DEPENDENT EFFECTS OF DOPAMINE ON JOINT INVASION IN ARTHRITIS}

L. Van $\mathrm{Nie}^{1}$, S. Rehart ${ }^{2}$, E. Neumann ${ }^{1}$, U. Müller-Ladner ${ }^{1}$, S. Capellino ${ }^{3}$. ${ }^{1}$ Department of Internal Medicine and Rheumatology, Justus-Liebig-University Gießen, Kerckhoff-Klinik, Bad Nauheim, Bad Nauheim; ${ }^{2}$ Department of Orthopedics and Trauma Surgery, Apaglesion Markus Hospital, Frankfurt am Main, Frankfurt am Main; ${ }^{3}$ Dept. of Immunology, Leibniz Research Centre for Working Environment and Human Factors, Dortmund, Germany

Background: Preventing synovial fibroblasts (SF) from migrating into the adjacent cartilage is a desirable therapeutic target in rheumatoid arthritis (RA) in order to avoid joint destruction and disability. In our previous studies we could show that RASF as well as SF from osteoarthritis (OA) patients express all dopamine receptor (DR) subtypes and dopamine stimulation alters proinflammatory cytokines (Capellino S et al, A\&R 2014).

Objectives: Therefore, we aimed to elucidate a potential dopamine-mediated impact on joint invasion and destruction in arthritis.

Methods: SF from RA and OA patients were obtained from patients undergoing knee joint replacement surgery (mean age: $74.3 \pm 11$.3yrs at OA and $73.7 \pm 10.3$ yrs at RA patients) to investigate dopamine receptor (DR)-distribution within the RA synovium and in the invasion zone, immunohistochemistry was performed for all five DR-subtypes. Migration and motility assays were performed under D1-like (D1DR and D5DR) and D2-like (D2DR, D3DR and D4DR) receptor stimulation. Dopamine effects on MMP3 and proMMP1 were evaluated using ELISA.

Results: D1DR, D4DR and D5DR were found to be stronger expressed close to the invasion zone and more cells were expressing the respective DR. Migration of RASF and OASF was significantly correlated with patients' age at surgery: younger patients ( $\leq 75$ years) showed an increase in migration up to $78 \%$ whereas older patients ( $\geq 75$ years) showed a reduced migration of up to $50 \%$ (OA $n=8$; RA $n=7$ ). There was no difference between RA and OA patients and between D1-like and D2-like receptor stimulation. The same effect could be observed in the motility assay (OA $n=5 ; R A n=6)$. MMP3 levels are altered under DR activation (OA $n=6 ;$ RA $n=6$ ).

Conclusions: The high DR expression close to the invasion zone suggests a direct role of dopamine on RASF aggressiveness and may contribute to cartilage invasion. This was confirmed in the in vitro assays, and supports the idea of a therapeutic potential of the dopamine pathway in RA.

Disclosure of Interest: None declared

DOI: 10.1136/annrheumdis-2017-eular.5266

\section{AB0070 CHONDROPROTECTIVE EFFECTS LINKED TO REDUCTION OF ADIPOGENESIS AND REGULATION OF GAP JUNCTION INTERCELLULAR COMMUNICATION: REGENERATIVE POTENTIAL THERAPEUTIC APPROACH FOR OSTEOARTHRITIS}

M. Varela Eirin ${ }^{1}$, A. Casado-Díaz ${ }^{2}$, J. Loureiro ${ }^{3}$, J.R. Caeiro ${ }^{3}$,

J.M. Quesada-Gómez ${ }^{2}$, M.D. Mayán ${ }^{1} .{ }^{1}$ Translational Research in Cell Communication and Signaling (CellCOM Research Group), Instituto de Investigación Biomédica de A Coruña (INIBIC). CH-Universitario A Coruña (XXIAC). Servizo Galego de Saúde (SERGAS). University of A Coruña, $A$ Coruña; ${ }^{2}$ Clinical Management Unit of Endocrinology and Nutrition, Maimónides Biomedical Research Institute of Córdoba (IMIBIC). Hospital Universitario Reina Sofía - RETICEF. Universidad de Córdoba, Córdoba: ${ }^{3}$ Department of Orthopaedic Surgery and Traumatology, Complexo Hospitalario Universitario de Santiago de Compostela (CHUS-XXIS). SERGAS, Santiago de Compostela, Spain

Background: Human bone marrow mesenchymal stem cells (hMSCs) exhibit an age-dependent reduction in osteogenesis and an increased propensity toward adipocyte differentiation. This switch has been associated with different bone disorders characterized by reduced bone formation and increased bone marrow fat accumulation. Connexin43 (Cx43) is an integral membrane protein that forms gap junction channels (GJs) and it is implicated in multiple cellular functions including cellular differentiation and control of bone remodelling and cartilage structure and function.

Objectives: In this study we investigated the effect of oleuropein and other molecules isolated from the Olea europaea in cellular differentiation to test if these molecules act as adipogenic suppressors in order to promote bone ad cartilage regeneration through a $\mathrm{Cx} 43$-dependent mechanism.

Methods: hMSCs were obtained from bone marrow donors. Human chondrocytes were isolated from cartilage of healthy donors and patients with osteoarthritis $(\mathrm{OA})$. Differentiation assays were carried out in the presence of different concentrations of oleuropein and olive extract (OE). Cellular differentiation was evaluated using histological stains. Scrape loading assays, western-blot, realtime qPCR and immunohistochemistry (IHC) assays were used to study the cellular communication through gap junction (GJs) and the levels and changes in subcellular localization of $\mathrm{C} \times 43$ and extracellular matrix (ECM) proteins such as Collagen type II (Col2)

Results: hMSCs treated with olive derived molecules showed a two-fold decrease in adipogenic differentiation, while osteogenesis and chondrogenesis were significantly increased. IHC, RT-qPCR and Western-Bot analysis of hMSCs supplemented with oleuropein/OE showed increased levels of Cx43. On the other hand, OA chondrocytes showed higher levels of Cx43 in comparison with normal chondrocytes; oleuropein and OE treatments significantly decreased Cx43 levels and dye transference through GJ channels. The treatment of $\mathrm{OA}$ chondrocytes micromasses with oleuropein and $\mathrm{OE}$ increased the levels of proteoglycans and Col2 in the extracellular matrix. These changes in the micromasses were accompanied by a decrease in $\mathrm{C} \times 43$ levels improving protein subcellular localization.

Conclusions: Together, our results suggest that the molecules used in this assays, via $\mathrm{C} \times 43$ and GJ intercellular communication increase the propensity towards osteogenesis and chondrogenesis, reducing adipocyte differentiation. Our assays indicate that these molecules may represent a potential therapeutic approach for cartilage and bone age-related disorders such as OA in order to promote cartilage regeneration.

Disclosure of Interest: None declared

DOI: 10.1136/annrheumdis-2017-eular.4830

\section{AB0071 ANALYSIS OF SYNOVIAL EXPRESSION OF PARVOVIRUS B19 ASSESSED IMMUNOHISTOCHEMICALLY AND CORRELATED TO SYNOVITIS SCORE}

M. Tarasovs ${ }^{1}$, A. Kadisa ${ }^{2}$, A. Mihailova ${ }^{3}$, S. Skuja ${ }^{4}$, M. Murovska ${ }^{5}$, V. Groma ${ }^{4}$ ${ }^{1}$ Riga Stradins University; ${ }^{2}$ Department of Internal Diseases, Riga East Hospital Clinic "Gailezers"; ${ }^{3}$ Orto Clinic; ${ }^{4}$ Institute of Anatomy and Anthropology; ${ }^{5} \mathrm{~A}$. Kirchenstein Institute of Microbiology and Virology, Riga Stradins University, Riga, Latvia

Background: Apart from systemic symptoms of viral infection parvovirus B19 (B19) could lead to acute and chronic arthropathy. It has been found in synovial tissue of rheumatoid arthritis (RA) patients, sometimes, being associated with some forms of undifferentiated arthritis. Possibly, it could promote inflammation in various forms of arthritis.

Objectives: To determine the expression of B19 antigens in different compartments of synovial membrane; correlate these findings to the estimated synovitis score.

Methods: 7 RA and 54 osteoarthritis (OA) patients were enrolled in this study. $\mathrm{nPCR}$ was used to detect the presence of B19 genomic sequence in 61 samples of synovial tissue. PCR B19 positive tissue samples were immunohistochemically treated with the anti-B19 antibodies detecting B19 capsid proteins' VP1/VP2 expression estimated thereafter semiquantitatively. The intimal and subintimal layers of synovium as well as vasculature were estimated. Synovial inflammation was evaluated using synovitis score.

Results: 3 RA and 3 OA patients were PCR B19 tissue positive. B19 antigens expression was observed in synovial lining, immune infiltrates and vascular endothelium. Correlation between B19 expression observed in synovial cells and inflammatory infiltrates' lymphocytes and macrophages was $r=0.555(p<0.0001)$ and $r=0.793(p<0.0001)$, respectively. Likewise, correlation between vascular endothelial B19 expression and synovial lymphocytic infiltrates was demonstrated $r=0.616(p<0.0001)$. Determined synovitis score varied from low up to intermediate revealing median value 2 . Simultaneously, there was no correlation found between the synovitis score and B19 antigens' expression.

Conclusions: B19 capsid proteins' VP1/VP2 expression is detectable in different structural constituents of synovial membrane. Under conditions studied, the tissue expression of B19 antigens does not correlate with the inflammatory indices scored.

Disclosure of Interest: None declared

DOI: 10.1136/annrheumdis-2017-eular.6411 